\title{
Deserción de la carrera
} de enfermería

\section{Autores:}

Jacqueline Lucía Ávalos Blaser. Enfermera, Docente Escuela Enfermería sede Viña del Mar, Universidad Santo Tomás, Chile. javalosb@santotomas.cl

María Graciela Flores Agüero. Enfermera. docente Escuela Enfermería sede Viña del Mar, Universidad Santo Tomás, Chile. mfloresa@santotomas.cl

Boris Paolo Moena González. Nutricionista docente Escuela Enfermería, sede Viña del Mar, Universidad Santo Tomás, Chile. borismoenago@santotomas.cl

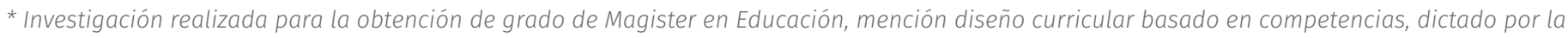
Escuela de Educación de la Universidad Santo Tomás, sede Viña del Mar.

\section{> Resumen}

Introducción. La deserción académica en el ámbito universitario, es un problema que enfrentan las instituciones de educación superior, se presenta a nivel nacional como internacionalmente y en establecimientos públicos como privados. Por ello, el objetivo de esta investigación fue identificar la tasa de deserción en los estudiantes de la carrera de Enfermería entre los años 2004 - 2012 de la Universidad Santo Tomás sede Viña del Mar. Material y Método. Estudio descriptivo, que incorporó a toda la población de estudiantes de la carrera de Enfermería que desertó en el periodo 2004-2012, de la Universidad Santo Tomás, sede Viña del Mar, se realizó un muestreo intencionado de 34 estudiantes. La información se recolectó a través de la aplicación de un cuestionario autoaplicado, diseñado por el investigador, denominado Deserción Viña I (DEVI-I); la información sobre el número de estudiantes desertores se obtuvo desde la base de datos interna de la Universidad (CLAS). Resultados. La tasa de deserción global de la carrera de Enfermería es de 5,27\%, ocurre durante los tres primeros años de la carrera. Conclusiones. La tasa global de deserción observada es inferior a la reportada en la misma universidad, o por otras carreras de Enfermería a nivel nacional, América Latina y países de la OCDE.

, Palabras clave: abandono escolar, universidades, enfermería (DeCS) 


\section{Dropping out of nursing school}

\section{, Abstract}

Introduction. School dropout in the university environment is a serious problem in higher education's institution today in national and international levels, and also in public \& private establishments. Objective. To establish the dropout rate among nursing students between 2004 and 2012 at Santo Tomas University, Viña del Mar. Material and Method. Descriptive Study, the sample included the entire student population of the Nursing career that deserted in the period 2004-2012, of the University Santo Tomas, Viña del Mar headquarters. The sampling was intentional, and corresponded to 34 students. The information of the general characteristics of the participants was collected through the application of a self-applied questionnaire, called Desertion Viña I (DEVI-I), designed by the researcher. The information on the number of student's deserters and enrolled were obtained from the CLAS system of the University. Results. The overall dropout rate of the Nursing career is 5,27\% average and it occurs during the first three years. Conclusions. The overall dropout rate of the Nursing career, presents values much lower than the overall rate of the same university and other nursing careers at the national level. The overall dropout rate of the Nursing career is much lower than the overall rate at the same university, other nursing careers at national and Latin American levels and OECD countries.

, Keywords: Student Dropouts, Universities, Nursing (DeCS). 


\section{) Introducción}

La deserción universitaria, por su magnitud, se ha convertido en un fenómeno muy común en la mayoría de los países, razón por lo cual se viene planteando como uno de los problemas prioritarios a ser investigado y adecuadamente tratado (1). El tema ha tomado un lugar importante en el debate público debido a que las pérdidas financieras y sociales que representan los estudiantes desertores son altas para la sociedad, para las instituciones de educación superior, las familias y el individuo. Junto con esto, el escaso conocimiento que se tiene en el país sobre los ciclos de la deserción, su adecuada forma de estudio y las políticas más efectivas para disminuirla, dificultarían aún más el trabajar en esta problemática.

Los resultados de las investigaciones plantean diferentes interrogantes sobre la incidencia del funcionamiento administrativo y pedagógico de las instituciones de educación superior (1), considerando que la problemática de la deserción no es tan sólo responsabilidad del estudiante, aunque se asume que gran parte de la responsabilidad recae sobre el desertor. El fenómeno de la deserción es inherente a la vida estudiantil, como consecuencia de problemas económicos, de interacción, socio-culturales del entorno y muchas más. En este sentido el fenómeno de la deserción persistirá de alguna manera hasta que cambien las instituciones universitarias. Por tanto, conocer el problema permitirá implementar estrategias dirigidas a establecer soluciones que controlen parcialmente los altos índices de deserción y de esa manera mantener una tendencia creciente en la retención de estudiantes.

La preocupación por la retención de estudiantes en la enseñanza superior chilena es un fenómeno más bien reciente, pese a que el fracaso estudiantil en la enseñanza universitaria ha sido consustancial a la existencia misma de las universidades, espe- cialmente de algunas carreras profesionales (2). La visualización de la retención es una temática en nuestro medio, donde aún no se comprende a cabalidad su impacto social y económico, ni se entiende en toda su magnitud la red de implicancias que se derivan del fracaso en los estudios en la enseñanza superior.

En Chile, hace algo más de una década, la preocupación por la retención de estudiantes en la educación superior comenzó a ser materia de significación, impelida esencialmente por la competencia de nuevos centros de enseñanza, la mayor escasez relativa de estudiantes, especialmente de aquellos en condiciones de pagar aranceles onerosos. En este contexto el aporte privado de las familias en la financiación de los estudios de educación superior en Chile supera en muchos casos el $50 \%$ del presupuesto regular de estos centros (2). Esto implica que la deserción en la educación superior se privatiza, tanto porque se asigna la responsabilidad casi absoluta al estudiante, como por la estructura de financiamiento, que descansa en el rol central de las familias. Es así como el análisis de la retención de estudiantes en la educación superior involucra un conjunto de dimensiones que atañen la eficiencia técnica de los procesos de formación (diseño curricular), la eficiencia económica y el impacto social.

En Latinoamérica se observa un 22\% de deserción en universitarios, mientras que en nuestro país esta tasa alcanza a un $19 \%$ en las universidades adscritas al Consejo de Rectores de Universidades Chilenas (CRUCH) y a un $22 \%$ en las universidades privadas autónomas $(3,4)$. La mayor proporción de titulados (retención) en Educación Terciaria en los países de la OCDE y en los de la Unión Europea, se da en los estudios de Grado o equivalente, con un $72,4 \%$ y un $71,3 \%$, respectivamente (5), por otra parte, la tasa de abandono de los estudios en el primer curso es del 19\% en los países de la OCDE cifra que es levemente inferior a los de Latino América 
y coincidentes con las universidades adscritas al CRUCH.

Las cifras de fracaso en Chile siguen siendo elevadas, afectando de manera evidente y significativa a los estudiantes de los dos primeros quintiles de ingresos (3), lo que contribuye decididamente a la reproducción de la desigualdad social, con el agravante, ya señalado, que muchas familias se han endeudado en cifras importantes para dar soporte financiero a los que estudian (6).

Así, la retención no solamente implica una dimensión de eficiencia de inversión técnica y económica, sino que incide en la equidad, al involucrar en mayor medida a la población de menores ingresos, por cuanto la condición de vulnerabilidad e inequidad en las oportunidades inciden en que la probabilidad de fracaso sea mayor para este grupo, como también de extender su ciclo formativo y de paso, postergar su ingreso al mercado laboral, lo que contribuye a un mayor endeudamiento, menor ingreso económico privado y mayor costo relativo de la formación.

Actualmente en el país, y pese a los avances señalados, se considera que el fenómeno en análisis no posee el reconocimiento público que amerita. Su magnitud real se desconoce, pues las cifras públicas al respecto son dificiles de comparar $(2,7,8)$.

Considerando esta problemática, la presente investigación se plantea como objetivo identificar la tasa de deserción en los estudiantes de la carrera de Enfermería entre los años 2004 - 2012 de la Universidad Santo Tomás, sede Viña del Mar.

\section{> Material y Método}

Esta investigación se plantea desde una visión positivista con enfoque cuantitativo a nivel descriptivo, de diseño no experimental y corte transversal.

La población incorporó a los estudiantes de la ca- rrera de Enfermería que desertaron en el periodo 2004-2012, de la Universidad Santo Tomás de la sede Viña del Mar. El muestreo fue intencionado y correspondió a 34 estudiantes, aunque cabe destacar que hubo pérdida de información, por lo que en algunas ocasiones se presenta información solo de 30 participantes.

Como criterios de inclusión se establecieron: ser estudiante que dio aviso de su retiro definitivo y voluntario de la carrera, ya sea en el primer o segundo semestre, entre el primer y quinto año de la carrera, de los años 2004 al 2012.

Entre los criterios de exclusión, se contemplaron: ser estudiante eliminado de la carrera por mal rendimiento académico, por medida disciplinaria, estudiante que no se matriculó en el programa de estudio, estudiante trasladado a otra sede, estudiante que hace retiro temporal o estudiante que se retiró sin previo aviso.

La variable principal de este estudio fue definida como la tasa de deserción de estudiantes de enfermería 2004-2012. Y como variables descriptivas se consideraron, edad, género, estado civil, tipo de establecimiento educacional de egreso de enseñanza media, nivel educacional del padre, nivel educacional de la madre, número de estudiantes desertores según año de carrera y número de estudiantes inscritos según año de carrera.

La información respecto a las características generales de los participantes, se recopiló a través de la aplicación de un cuestionario autoaplicado, diseñado por la investigadora principal, denominado Deserción Viña I (DEVI-I), conformado por 7 ítems con respuestas de selección múltiple y respuestas abiertas. El tiempo de aplicación fue de 5 minutos por persona.

Se contempló un análisis descriptivo y multivariado para establecer la estructura del instrumento. 
La confiabilidad del mismo se realizó mediante un análisis de confiabilidad basado en la teoría clásica de análisis de ítems y pruebas de hipótesis de relación (Test Chi-Cuadrado) y de comparación de proporciones (Test de comparación de proporciones) para apoyar las conclusiones.

Los datos fueron analizados por el software estadístico SPSS versión 18.0. Los datos continuos y ca- tegóricos se presentan como frecuencias, porcentajes y tasas.

Al inicio de esta investigación se contó con la autorización de la Dirección Académica y de la Dirección de la Carrera de Enfermería de la institución, contempló los requisitos éticos de E. Emmanuel (9). Todos los participantes firmaron consentimiento informado previo a responder el instrumento.

\section{) Resultados}

TABLA 1. CARACTERÍSTICAS SOCIODEMOGRÁFICAS DE LOS ESTUDIANTES DESERTORES DE LA CARRERA DE ENFERMERÍA 2004-2012, UST, SEDE VIÑA DEL MAR

\begin{tabular}{|c|c|c|c|}
\hline VARIABLE & CATEGORÍAS & $\mathbf{f}$ & $\%$ \\
\hline \multirow[t]{3}{*}{ Edad } & 18 - 21 Años & 9 & 30,0 \\
\hline & 22 y más Años & 21 & 70,0 \\
\hline & Total & 30 & 100 \\
\hline \multirow[t]{3}{*}{ Género } & Masculino & 5 & 16,7 \\
\hline & Femenino & 25 & 83,3 \\
\hline & Total & 30 & 100 \\
\hline \multirow[t]{5}{*}{ Estado civil actual } & Casado(a) & 4 & 13,3 \\
\hline & Soltero(a) & 25 & 83,3 \\
\hline & Divorciado(a) & 1 & 3,3 \\
\hline & Viudo(a) & 0 & 0,0 \\
\hline & Total & 30 & 100 \\
\hline \multirow[t]{5}{*}{ Establecimiento educacional de egreso de enseñanza media } & Municipalizado & 5 & 16,7 \\
\hline & Particular Subvencionado & 21 & 70,0 \\
\hline & Particular & 4 & 13,3 \\
\hline & Otro & 0 & 0,0 \\
\hline & Total & 30 & 100 \\
\hline \multirow[t]{9}{*}{ Nivel educacional del padre } & Enseñanza Básica completa & 1 & 3,7 \\
\hline & Enseñanza Básica incompleta & 0 & 0,0 \\
\hline & Enseñanza Media completa & 11 & 40,7 \\
\hline & Enseñanza Media incompleta & 0 & 0,0 \\
\hline & Educación Superior Técnica completa & 5 & 18,5 \\
\hline & Educación Superior Técnica incompleta & 0 & 0,0 \\
\hline & Educación Superior Profesional Completa & 9 & 33,3 \\
\hline & Educación superior Prof. Incompleta & 1 & 3,7 \\
\hline & Total & 27 & 100 \\
\hline \multirow[t]{9}{*}{ Nivel educacional de la madre } & Enseñanza Básica completa & 1 & 3,3 \\
\hline & Enseñanza Básica incompleta & 2 & 6,7 \\
\hline & Enseñanza Media completa & 16 & 53,3 \\
\hline & Enseñanza Media incompleta & 3 & 10,0 \\
\hline & Educación Superior Técnica completa & 5 & 16,7 \\
\hline & Educación Superior Técnica incompleta & 2 & 6,7 \\
\hline & Educación Superior Profesional Completa. & 1 & 3,3 \\
\hline & Educación superior Prof. Incompleta & 0 & 0,0 \\
\hline & Total & 30 & 100 \\
\hline
\end{tabular}


Al observar la tabla 1, que muestra las características de los estudiantes de Enfermería que desertaron entre el periodo 2004 -2012, destaca que el 70,0\% tiene 22 y más años, el 83,3\% pertenecen al género femenino, el $83,3 \%$ son solteros y el $70,0 \%$ realizó su enseñanza media en un establecimiento particular subvencionado.

En relación al nivel educacional de los padres, el 40,7\% tiene la Enseñanza Media completa y un 33,3\% de los padres tiene Educación Superior Profesional Completa. En cuanto al nivel educacional de las madres un 53,3\% tiene la Enseñanza Media completa y sólo el 3,3\% tiene Educación Superior Profesional Completa.

TABLA 2. ESTUDIANTES DESERTORES SEGÚN AÑO QUE CURSABAN EN LA CARRERA DE ENFERMERÍA, UST SEDE VIÑA DEL MAR 2004-2012.

\begin{tabular}{|l|r|r|}
\hline Año de carrera & f & \% \\
\hline Primer año & 12 & 35,3 \\
\hline Segundo año & 8 & 23,5 \\
\hline Tercer año & 14 & 41,2 \\
\hline Cuarto año & 0 & 0,0 \\
\hline Quinto año & 0 & 0,0 \\
\hline Total & $\mathbf{3 4}$ & $\mathbf{1 0 0}$ \\
\hline
\end{tabular}

En la tabla 2 se aprecia que el año o nivel de la carrera que presenta la mayor tasa de deserción es en tercer año, correspondiendo a un $41,2 \%$, seguido por el primer año con un 35.3\% y segundo año con $23,5 \%$. Otro aspecto que es importante destacar es que durante el cuarto y quinto año no se observa deserción.
TABLA 3. TASA DE DESERCIÓN POR AÑO, PERÍODO 2004-2012, CARRERA DE ENFERMERÍA UST, SEDE VIÑA DEL MAR.

\begin{tabular}{|l|r|r|r|}
\hline Año & $\begin{array}{r}\text { Población de } \\
\text { estudiantes } \\
\text { desertores por año }\end{array}$ & $\begin{array}{r}\text { Alumnos Inscritos } \\
\text { por año }\end{array}$ & Tasa \\
\hline $\mathbf{2 0 0 4}$ & 2 & 64 & 3,1 \\
\hline $\mathbf{2 0 0 5}$ & 2 & 51 & 3,9 \\
\hline $\mathbf{2 0 0 6}$ & 2 & 54 & 3,7 \\
\hline $\mathbf{2 0 0 7}$ & 0 & 63 & 0,0 \\
\hline $\mathbf{2 0 0 8}$ & 4 & 64 & 6,3 \\
\hline $\mathbf{2 0 0 9}$ & 2 & 63 & 3,2 \\
\hline $\mathbf{2 0 1 0}$ & 1 & 60 & 1,7 \\
\hline $\mathbf{2 0 1 1}$ & 14 & 81 & 17,3 \\
\hline $\mathbf{2 0 1 2}$ & $\mathbf{7}$ & 84 & 8,3 \\
\hline Total & $\mathbf{3 4}$ & $\mathbf{5 8 4}$ & $\mathbf{5 , 3}$ \\
\hline
\end{tabular}

La tabla 3 muestra que la tasa de deserción se comporta de manera uniforme entre los años 2004 al 2012, excepto el 2011, que representa el año con mayor porcentaje de deserción, correspondiendo a un 17,3\%, sigue el año 2012 con un 8,3\% y el año 2008 con un 6,3\%. Llama la atención el año 2007 donde no hubo deserción.

\section{, Discusión}

A la luz de los resultados de este estudio se observa que la tasa de deserción global de la carrera de Enfermería en la UST Sede Viña del Mar, durante el periodo 2004-2012, es de 5,3\%, cifra que resulta estar muy por debajo de los valores mostrados por la realidad chilena (11,0\%) (10), por la misma UST $(22,0 \%)$, e incluso son menores que la deserción mostrada por las carreras de Enfermería de las universidades en Chile $(11,4 \%)$ (11). Estos resultados podrían deberse a la eficiencia de titulación mostrada por las carreras del área de la salud (12).

En relación a las características socioeducativas de los estudiantes desertores de la carrera de enfermería destaca que la edad de 22 años es donde se encuentra el mayor porcentaje de desertores, correspondiendo a un $70 \%$, esto concuerda con lo 
encontrado por otros investigadores que señalan que entre los 22 y 23 años es el promedio de edad al momento de la deserción (3,13-15). Esto se explica básicamente porque la edad promedio de ingreso de los jóvenes a las universidades chilenas es de alrededor de 18 años (16), por otra parte, no todos los estudiantes encuestados van al día con sus estudios, por diversas causas, y además este puede haber cursado una carrera previa

Respecto al género, el 83,3\% de los desertores corresponde al género femenino. Este resultado coincide con un estudio realizado en la carrera de Enfermería de una universidad del Ecuador, donde un $85,4 \%$ de los estudiantes que desertaban también eran mujeres (17), Esta situación se produce dado que Enfermería es una carrera eminentemente femenina, aunque estos últimos años se ha ampliado a los varones.

El 83,3\% de los estudiantes desertores es soltero, cifras que coinciden con un estudio colombiano, realizado en la carrera de Enfermería (4). Por otro lado, el Censo 2012 arrojó que el 47,23\% de la población se encuentra soltera, además muestran que la edad media de los contrayentes al momento de casarse en el país es de 35,4 años para los hombres y de 32,7 años en las mujeres (18), lo que explicaría el alto porcentaje de solteros entre los estudiantes desertores.

Los estudiantes que abandonan la carrera en su mayoría egresan de un establecimiento particular subvencionado, cifra que coincide con las reportadas por el Ministerio de Educación de Chile (MINEDUC) donde se menciona que un $54,6 \%$ de los jóvenes egresan de este tipo de establecimientos (19), valor que se repite también al comparar con los demás estudiantes de la UST, donde el 95,0\% proviene de colegios municipalizados o particulares-subvencionados (20).
Otra de las características medida en este grupo es el nivel de educación de los padres, esta variable que puede influir en los factores que determinan la deserción, especialmente, el de la madre, en razón que, a mayor nivel de educación de los padres, se presenta una menor deserción $(4,21)$. Así, tenemos que el $40,7 \%$ de los padres tiene enseñanza media completa y un 33,3\% educación superior profesional completa. En cuanto al nivel educacional de las madres, un 53,3\% tiene enseñanza media completa y sólo el 3, 3\% tiene educación superior profesional completa. Cifras muy similares a las encontradas en investigaciones realizadas a estudiantes de las carreras de enfermería y kinesiología en otras universidades chilenas (21-23).

Por su parte, las cifras sobre retención en el sistema, indican que un significativo número de estudiantes abandona su carrera en primer o segundo año, disminuyendo la proporción de estudiantes que desertan a partir de tercer año $(10,24)$. En este sentido, la tasa de deserción para los cursos de primero, segundo y tercer año de la carrera de enfermería UST, se comporta de manera diferente a lo expuesto, donde la mayor deserción se produce en el tercer año, con un 41,2 \%, luego primer año con un $35,3 \%$ y finalmente segundo año con $23,5 \%$. Este hecho podría explicarse porque en este nivel (tercer año) se comienza intensivamente con las prácticas curriculares de manera simultánea con la cátedra, es decir, se cuenta con un semestre (18 semanas) dividido entre clases, prácticos de laboratorio, seminarios y experiencia clínica, con la consiguiente carga emocional y psicológica. Sumado a esto, probablemente la falta de hábitos de estudio de estos jóvenes y la falta de preparación para enfrentar las exigencias universitarias, (un gran porcentaje de alumnos proviene de colegios particulares subvencionados y municipales) (25), pueden ser factores involucrados en esta temáti- 
ca. Para el cuarto y quinto año no hubo estudiantes desertores, debido probablemente al avance curricular y consolidación vocacional.

Además, al comparar la deserción en primer y segundo año, se aprecia que estos valores son mayores respecto a la tasa global de la UST, donde se presenta un $22 \%$ en primer año y un $37 \%$ en segundo año. No se disponen de datos de la UST para tercer, cuarto y quinto año, por lo que no es posible comparar en estos niveles.

Así mismo, al comparar la deserción entre enfermería de la UST y las medias de deserción en las carreras de enfermería en Chile también existen diferencias, mostrándose tasas más altas en la UST en los tres primeros años. Así, en el primer año de enfermería UST se presenta un 35,3\% a diferencia de $11,0 \%$ promedio en enfermería nacional, luego en el segundo año $23,5 \%$ versus un $16,0 \%$ de promedio en enfermería nacional; en seguida, tercer año de enfermería UST duplica su valor a 41,2\% a diferencia de enfermería nacional con $20 \%$ promedio. Luego al comparar el cuarto y quinto año se puede advertir que no se producen deserciones a este nivel en enfermería UST, a diferencia de enfermería nacional que mantiene un $23,0 \%$ y un $26,0 \%$ promedio de deserción respectivamente (5).

Ahora bien, al determinar la tasa de deserción por año entre el año 2004 y 2012, se puede observar que se comporta de manera uniforme en este periodo, excepto el 2011, que representa el año con mayor porcentaje de deserción con un 17,3\%. Cabe destacar que ese año aumentó el número de vacantes para el primer año de 64 a 81 en la carrera de enfermería (en aproximadamente $26 \%$ ), hecho que podría haber sido causal del aumento en la deserción.

Con todo lo anterior, es posible concluir que la tasa de deserción global de enfermería UST sede Viña del Mar para el periodo 2004-2012 presenta valores muy inferiores respecto a la tasa global UST y Enfermería a nivel nacional e internacional. Las tasas de deserción más altas se presentan en los estudiantes de tercer y primer año de la carrera.

Los estudiantes de Enfermería que desertaron tienen en promedio 22 años, son de género femenino, solteros, en su mayoría egresaron de un establecimiento particular subvencionado. Respecto al nivel educacional de ambos padres, casi la mitad tiene Enseñanza Media completa y un tercio de los padres tiene Educación Superior Profesional Completa, no así sus madres que tienen muy bajo porcentaje de Educación Superior Profesional Completa. Estas características se repiten en numerosos estudios relacionados con los factores de riesgo de deserción o factores que explican la deserción de los estudiantes universitarios.

Finalmente, el problema de la deserción universitaria debería ser abordado desde distintos focos, ya que es un fenómeno en el cual interactúan distintos factores con diversas implicancias (12). Se considera necesario desarrollar investigaciones que permitan profundizar en diferentes aristas para abordar esta problemática integralmente.

\section{Referencias bibliográficas}

1. Saldaña M, Barriga OA. Adaptación del modelo de deserción universitaria de Tinto a la Universidad Católica de la Santísima Concepción. Revista de ciencias sociales. 2010; 16(4): 616-28.

2. Organización para la Cooperación y el Desarrollo Económicos, Banco Mundial. La Educación Superior en Chile. Santiago de Chile: Ministerio de Educación de Chile; 2009. 329p.

3. Jiminián YY. Deserción en la Universidad abierta para adultos (UAPA), durante el periodo 
2001-2006. [Tesis Doctoral] Santiago: República Dominicana. Universidad Estatal a Distancia, 2002.

4. Piratoba BN, Ovidio Ch. Factores de deserción de los estudiantes en la facultad de enfermería de la Universidad de ciencias aplicadas y ambientales U.D.C.A, durante el periodo: 2009-2010-I 2011. Rev UDCA Act y Div Cient. 2013 (jul-dic); 16(2): 553-62.

5. Ministerio de Educación, Cultura y Deporte de España. Panorama de la educación. Indicadores de la OCDE 2016. Informe Español. Madrid (España); 2016.

6. Donoso S, Cancino V. Caracterización socioeconómica de los estudiantes de educación superior. Calidad en la educación. 2007; 26: 203-44.

7. Centro Interuniversitario de Desarrollo (CINDA). Repitencia y deserción universitaria en América Latina. Santiago (Chile): CINDA, UNESCO; 2006. 380p.

8. Ministerio de educación de Chile. Serie Evidencias: Deserción en la educación superior en Chile. [Internet] 2012. [acceso 10 jun 2017]; 1(9): Disponible en: http://portales.mineduc.cl/usuarios / bmineduc/doc/201209281737360.EVIDENCIASCEM9. pdf

9. Gaudlitz M. Reflexiones sobre los principios éticos en investigación biomédica en seres humanos. Rev chil enferm respir. [Internet] 2008 [acceso jun 2017]; 24 (2): 138-42. Disponible en: http:// www.scielo.cl/scielo.php?script=sci_arttext $\&$ pid= S0717-73482008000200008

10. Consejo Nacional de Educación (CNED), Departamento de Investigación e Información Pública. Indicadores, números y datos sobre instituciones y carreras de educación superior, INDICES. CNED, Ministerio de Educación de Chile. [Internet] 2010. [acceso jul 2017], Disponible en: http://cned. $\mathrm{cl} /$ public/Secciones/Seccion IndicesPostulantes/ Publicaciones/16_Metodolog\%C3\%ADaINDICESInd icadores_17082016.pdf

11. Consejo Nacional de Educación (CNED), Departamento de Investigación e Información Pública. Estadísticas de retención, INDICES. CNED, Ministerio de Educación de Chile. [Internet] 2010. [acceso jul 2017], Disponible en: http://www.cned. $\mathrm{cl} /$ public/Secciones/SeccionIndicesEstadisticas/ indices_estadisticas_retencion.aspx

12. González L, Uribe D. Estimaciones sobre la repitencia y deserción en la educación superior chilena. Consideraciones sobre sus implicancias. Calidad en la educación 2005; (17): 75-90.

13. Sanabria H. Deserción en estudiantes de enfermería en cuatro universidades del Perú. Anales de la Facultad de Medicina. 2002; 63(4): 301-11.

14. Ariza SM, Marín DA. Factores intervinientes en la deserción escolar de la Facultad de Psicología, Fundación Universitaria Los Libertadores. Tesis Psicológica. 2009; 4: 72-85.

15. Condori MP. Estrés académico y su relación con las estrategias de afrontamiento de los estudiantes de enfermería de la Universidad Nacional Jorge Basadre Grohmann. Tacna. [Internet] 2012. [acceso may 2017]; Disponible en: http://200.37.105.196:8080/ bitstream/handle/unjbg/170/55_2013_Condori_Condori_MP_FACS_Enfermeria_20013_Resumen.pdf?sequence $=2$

16. Universidad de Chile. Compendio estadístico proceso de admisión año académico 2009. [Internet] 2010 [acceso 01 jun 2017]; Disponible en: http://psu.demre.cl/estadisticas/documentos/ p2009/2009-compendio-estadistico.pdf

17. Yaselga B. Yépez S. Factores que intervienen en la deserción de los estudiantes de segundo y cuarto semestres de la Carrera de Enfermería, 
Facultad Ciencias de la Salud, Universidad Técnica del Norte. Periodo académico septiembre 2009agosto 2010. [Tesis de grado]. Ibarra, Colombia: Universidad Técnica del Norte 2010.

18. Instituto Nacional de Estadísticas de Chile (INE). Estadísticas vitales. [Internet] INE; 2010. [acceso jun 2017]. Disponible en: http:/ / www.ine.cl/

19. Ministerio de Educación de Chile (MINEDUC). Variación de matrícula y tasa de permanencia por sector. [Internet] MINEDUC; 2010. [acceso may 2017]. Disponible en: http://centroestudios. mineduc.cl/tp_enlaces/portales/tp5996f8b7 cm96/uploadImg/File/Evidencias/Evidencias\%20 final_julio_2015.pdf

20. Universidad Santo Tomás. Proceso de enseñanza y aprendizaje. En: Resumen ejecutivo de la evaluación institucional UST 2014-2017. Santiago de Chile; 2017.

21. Celis R, Flores CL, Reyes MC, Venegas $\mathrm{H}$. Factores de riesgo de deserción presentes en alumnos repitentes de las carreras de enfermería y kinesiología en una universidad chilena. Cienc enferm. 2013; 19(3): 63-71.

22. Román M. Factores asociados al abandono y la deserción escolar en América Latina: una mirada en conjunto. Revista iberoamericana sobre calidad, eficacia y cambio en Educación. [Internet] 2013. [acceso may 2017]; 11(2): 33-59. Disponible en: http:/ / www.redalyc.org/pdf/551/55127024002.pdf

23. Larroucau T. Estudio de los factores determinantes de la deserción en el sistema universitario chileno. Rev est de políticas públicas. [Internet] 2015. [acceso jun 2017]; 1:1-23. Disponible en: http:/ /dx.doi.org/10.5354/0719-6296.2015.38351

24. Himmel E. Modelos de análisis de la deserción estudiantil en la educación superior. Calidad en la educación. 2002; 17(2): 91-108.
25. Canales A, De los Ríos D. Factores explicativos de la deserción universitaria. Calidad en la educación. 2007; 26: 173-201. 